This item was submitted to Loughborough's Research Repository by the author.

Items in Figshare are protected by copyright, with all rights reserved, unless otherwise indicated.

\title{
Sleep duration and all-cause mortality: links to physical activity and prefrailty in a 27-year follow up of older adults in the UK
}

PLEASE CITE THE PUBLISHED VERSION

https://doi.org/10.1016/j.sleep.2018.11.008

\section{PUBLISHER}

(c) Elsevier

\section{VERSION}

AM (Accepted Manuscript)

\section{PUBLISHER STATEMENT}

This paper was accepted for publication in the journal Sleep Medicine and the definitive published version is available at https://doi.org/10.1016/j.sleep.2018.11.008.

\section{LICENCE}

CC BY-NC-ND 4.0

\section{REPOSITORY RECORD}

Morgan, Kevin, and luliana Hartescu. 2019. "Sleep Duration and All-cause Mortality: Links to Physical Activity and Prefrailty in a 27-year Follow up of Older Adults in the UK". figshare. https://hdl.handle.net/2134/36877. 
Sleep duration and all-cause mortality: links to physical activity and prefrailty in a 27-year follow up of older adults in the UK.

Kevin Morgan, $\mathrm{PhD}^{1}$

Iuliana Hartescu, $\mathrm{PhD}^{1}$

${ }^{1}$ Clinical Sleep Research Unit, School of Sport, Exercise and Health Sciences,

Loughborough University, Leicestershire, UK, LE11 3TU

Corresponding author: Iuliana Hartescu, Clinical Sleep Research Unit, School of Sport, Exercise and Health Sciences, Loughborough University, Leicestershire, UK, LE11 3TU Email: i.hartescu@lboro.ac.uk

Key words: sleep duration; mortality; insomnia; sedentary behavior; physical activity; frailty 
Objectives: To assess sleep duration-mortality relationships across a 27-year follow-up period in a well characterized random sample of older people, and to test the hypothesis that mortality risks associated with long sleep duration confound with, and can be explained by, low levels of functional capacity indicative of frailty.

Methods: Face-to-face interviews conducted among 1002 randomly sampled older (65+) people in 1985 provided baseline profiles of health, functional capacity, physical activity, and sleep quality and duration. Health and functional status in each of 6 sleep duration categories $(\leq 4,5,6,7,8, \geq 9$ hours) was examined. At censorship in 2012,927 deaths were recorded. Relationships between sleep duration and 27-year all-cause mortality were then examined in a series of incrementally adjusted Cox regression models.

Results: Associations between sleep duration and measures of sleep quality were predominantly linear, with longer sleep times indicating superior sleep quality. Relationships between sleep duration and functional capacity, on the other hand, were predominantly quadratic, with most approximating a U-shaped function. Adjusted for age, gender, social class, insomnia symptoms, physical health, depression, BMI and smoking status, long sleep duration and continuous hypnotic drug use at baseline were significantly and independently associated with elevated mortality risk (HR: 1.37; 95\% Cl: 1.05-1.78; HR: 1.24; $95 \% \mathrm{Cl}$ : 1.01-1.51). When indices of frailty were added to the model, hazard ratios for long sleep duration and hypnotic drug became non-significant, while the lowest physical activity quintile and very slow walking speed significantly increased mortality risk (HR: $1.79 ; 95 \% \mathrm{Cl}: 1.40-$ 2.30; HR: 1.41; 95\% Cl: 1.15-1.73 respectively).

Conclusions: In analyses of sleep-related mortality outcomes long sleep durations confound with, and may be indicative of, incipient frailty among older participants. 


\section{Introduction}

Subjectively reported long and short sleep durations have been consistently associated with increased all-cause mortality (e.g. Kripke et al, 2002; Patel et al, 2004; Ferrie et al, 2007; Ikehara et al, 2009; Castro-Costa et al, 2011; Kakizaki et al, 2013; Chen, Su \& Chou, 2013; Aurora et al, 2016), with meta analyses (Gallicchio and Kalesan, 2009; Cappuccio et al, 2010) suggesting a U-shaped sleep duration-mortality function. However, this U-shaped function has been reported mainly in studies where participants are asked to estimate an average or 'typical' total sleep time (TST). Studies where TST has been calculated (by the investigator) from reported bedtimes, rise times, and sleep latencies have tended to show a stronger relationship between long sleep duration and mortality (Kurina et al, 2013). It is likely that 'normative bias' (where participants in good health are inclined to report socially 'normal' sleep durations and vice-versa) is reduced using the latter method (Kurina et al, 2013). While longer sleep durations have been associated with an increased risk of incident diabetes, cardiovascular disease, stroke, coronary heart disease, and obesity (Jike et al, 2018), causal pathways linking longer TSTs to mortality risk remain under researched (Knutson \& Turek, 2006; Grandner \& Drummond, 2007; Patel et al, 2012).

Longer sleep durations could influence health and survival through the independent risks associated with sedentary behavior (see Thorp et al, 2011) and inactive lifestyles (see Samitz et al, 2011) since: a) longer sleep times, of necessity, reduce the time available for active behavior; and b) longer sleep times logically demand an extended time in bed (TIB) which may attract the same health risks as persistent sedentary behavior. Evidence consistent with these assumptions is provided by: the Nurses' Health Study II (Patel et al, 2006), which found longer sleep durations associated with both mortality and lower activity levels; the National Health Interview Survey which found both higher activity levels associated with a lower risk of long (but not short) sleep durations (Krueger \& Friedman, 2009), and that increased stroke prevalence associated with long (relative to short) sleep durations was mitigated by regular moderate physical activity (Seixas et al, 2018); and the 
combined Swedish Male and Swedish Mammography cohorts study (Bellavia et al, 2014) which found that long sleep durations were significantly associated with all-cause mortality in the least physically active sub-group. None of these studies, however, reported analyses which controlled for TIB.

Similarly emphasizing physical activity, the emergence of frailty in later life may also contribute to long sleep-mortality relationships (Lee et al, 2014; 2017). Defined as "...a state of vulnerability to poor resolution of homoeostasis after a stressor event" (Clegg et al, 2013), frailty does not necessarily reflect active disease processes, but is indexed rather to the cardinal signs of: self-reported exhaustion; low physical activity; slow walking speed; muscle weakness (as indicated by grip strength); and shrinking/weight loss (Afilalo et al, 2014). Both frail (where any 4 of these signs co-occur) and 'prefrail' (where any 2 of these signs cooccur) states have been shown to be predictive of mortality in models adjusted for age and self-reported mental and physical health status (Fried et al, 2001). Given this, longer sleep durations (and consequently longer TIBs) could reflect signs of 'exhaustion' and 'low physical activity' indicative of prefrail states in otherwise healthy individuals. Consistent with this, longer TIBs have been shown to significantly increase the risk of subsequent cognitive (Gabelle et al, 2017) and physical (Stenholm et al, 2011) decline in older people.

In the present analyses we combine detailed indices of functional capacity with established health and demographic covariates in models designed to evaluate sleep duration-mortality relationships across one of the longest follow-up periods yet reported for a random sample of older people. In addition, the analyses aimed: 1) to examine the longitudinal stability of survey-assessed TST categories; and 2) to characterize functional capacity, health and sleep quality profiles across sleep duration categories.

\section{METHODS}

Data were derived from the Nottingham Longitudinal Study of Activity and Ageing (NLSAA), full details of which are presented elsewhere (Morgan, 1998). Briefly, the NLSAA is an 
ongoing study of health outcomes among people originally aged 65 and over living in the UK. The baseline survey for the NLSAA was conducted between May and September 1985, during which time 1042 people, randomly sampled from the community, were interviewed in their own homes. Follow-up surveys were conducted in 1989 and 1993, with re-interview rates of $88 \%$ and $73 \%$ respectively obtained among survivors. Information on mortality within the baseline sample was provided by the UK Medical Research Information Service and handled by KM and IH as accredited Office of National Statistics (ONS) Approved Researchers. Survey waves and subsequent analyses were approved by relevant ethical agencies. Survey assessments relevant to the present analyses are described below.

\section{$\underline{\text { Physical activity }}$}

Customary physical activity (CPA) was assessed in 6 domains: indoor; outdoor; leisure; walking; strength; and flexibility. Continuous activities performed for at least 3 minutes/week for at least the previous six weeks were aggregated into total minutes/week. Prior to the assessment of walking participants were asked "Which of the following best describes your (typical) walking speed?", with the options: very slow; stroll at an easy pace; normal speed; fairly brisk; and fast. Non-continuous activities likely to contribute to muscle strength or joint flexibility were assessed in terms of frequency of performance. Principal components analysis of activity in all 6 domains extracted factor scores providing valid and reliable summary measurements of physical activity in this population (Morgan, 1998; Hartescu, Morgan \& Stevinson, 2015). For the survival analyses, activity was coded as a categorical variable using CPA1 quintile ranges to define low, medium low, medium, medium high, and high (reference) customary physical activity levels.

\section{Total Sleep Time (TST) and Time in Bed (TIB)}

Sleep duration and TIB were calculated from the items: 1) "At what time do you usually go to bed?"; 2) "At what time do you usually settle down in bed to go to sleep?"; 3) How long does it normally take you to fall asleep?"; 4) "At what time do you finally wake up?"; and 5) "At what 
time do you usually get up?" (TST = TIB minus all non-sleep time in bed). Sleep duration (TST) values were categorized in 1-hour units and rounded to the nearest whole number ranging from $\leq 4$ hours to $\geq 9$ hours (after Chen et al, 2013). Time in bed (TIB = time elapsed between going to bed and getting up) was similarly estimated in whole hours ranging from $\leq 6$ hours to $\geq 10$ hours. In common with previous studies (e.g. Kripke et al, 2002; Youngstedt and Kripke, 2004; Chen et al, 2013) the TST reference category was 7 hours. The TIB reference category was 8 hours.

\section{$\underline{\text { Insomnia symptoms }}$}

Sleep quality was assessed using the item "Do you ever have problems sleeping (i.e. problems getting to sleep and/or staying asleep and/or waking too early?)", with five response categories (never, seldom, sometimes, often, all the time). Positive responses were followed with the question "Have you had this problem/these problems in the past week?". Insomnia symptoms were considered present if the participant reported a sleep problem "often" or "all the time", and if that problem had been experienced within the previous week. The incidence and prevalence of insomnia symptoms assessed using these items closely matched epidemiological expectations (see Morgan, 2003).

\section{Muscle strength}

Maximum handgrip strength (in newtons) was assessed using a purpose-designed isometric dynamometer (Bassey, 1998). The highest value from 3 maximal attempts using either hand was used as the definitive measurement. Values in the lowest gender-specific quintile were categorized as 'frail'; all other values were categorized as 'non-frail'.

\section{$\underline{\text { Weight and Skeletal Size }}$}

At interview weight measurements (fully clothed with shoes removed) were made using calibrated analogue scales. To reduce error arising from kyphotic changes in measuring the height of older people, skeletal size was assessed using demi span, the distance between the finger roots and the sternal notch (with the arm laterally outstretched). BMI was then estimated 
using weight and predicted height (Lehmann et al, 1991) and categorized: $<18.5$ (underweight); $>18.5$ to $<25$ (healthy); $\geq 25$ overweight/obese.

\section{$\underline{\text { Hypnotic drug use }}$}

Prescription hypnotic drug use was assessed with the item "Do you take prescribed medicines to help you sleep". Reported hypnotic use was verified by the interviewer who examined tangible evidence of prescribed medication. Responses were categorized: continuous user; occasional user; and non-user.

\section{Physical Health Status}

General physical health was assessed using a health index scored from zero (low health burden) to 13 (high health burden) covering the presence or absence of: heart, stomach, eyesight, or foot problems; giddiness, headaches, urinary incontinence, arthritis, falls, and chronic disease; drug use (excluding hypnotics) and walking aid use, and recent (1 month) contact with (primary and secondary care) medical services. Smoking status (smoker v non-smoker) was assessed in a separate item.

\section{$\underline{\text { Mental Health Status }}$}

Affective status was assessed using the Symptoms of Anxiety and Depression (SAD) Scale (Bedford et al, 1976). Clinically significant levels of depressive symptoms are indicated by scores of $\geq 6$. At baseline, scores at or above this cut-point showed high levels of concordance with clinical diagnostic ratings of depression (Morgan 1998).

\section{$\underline{\text { Social Class }}$}

Social/occupational class (Elias et al, 1993) was based on main occupation (or husband's main occupation) before retirement, and collapsed into 3 categories: 1 (professional/managerial); 2 (skilled manual/non-manual); and 3 (semi-/unskilled manual).

\section{Statistical analyses}


Indices of sleep quality (including time spent in bed and sleep efficiency), physical activity, and physical capabilities (handgrip strength and shoulder flexibility) across the reported sleep duration categories were examined in regression models to test for linear and quadratic relationships. Associations between baseline sleep duration category and the prevalence of insomnia symptoms and hypnotic drug use were assessed using chisquare. Concordance between membership of baseline and subsequent 4- and 8-year follow-up sleep duration categories was assessed using Cohen's kappa.

Relationships between reported sleep durations and all-cause mortality were examined in four separate Cox regression models with days from baseline interview (a date between May 1st - September 30th, 1985) to death/censorship (9th February 2012) as dependent. In the first model we adjusted only for age and gender (Model 1). Two further models then incrementally adjusted for: social class; hypnotic drug use; insomnia symptoms; physical health; depression; BMl; and smoking status (Model 2); and physical activity quintile; very slow walking speed; frail handgrip strength; and TIB category (Model 3). Interaction terms (physical activity by sleep duration, physical activity by gender, and physical activity by time in bed) initially added to Models $3 \& 4$ were non-significant, and omitted from the final models. In all models age was entered as a categorical variable for the 5-year bands (65$69 ; 70-74 ; 75-79 ; \geq 80)$. To formally test the proportional hazards $(\mathrm{PH})$ assumption Schoenfeld's residuals were calculated for each covariate and regressed against survival time; results showed no significant departures from the $\mathrm{PH}$ assumption. To address issues of possible reverse causality (where health status at baseline might influence both sleep variables and mortality hazard), Models $1-3$ were repeated omitting deaths recorded within 3 years of the baseline interview. A forced entry approach to model selection was used throughout. All data were analyzed using SPSS for Windows v23.0.

\section{RESULTS}


From the baseline sample of 1002, 42 participants were omitted due to incomplete activity data $(n=14)$, incomplete anthropometric data $(n=12)$, or withholding permission for this element of the study $(n=18)$. In the 27 -year period $1985-2012$ the project received notification of 927 deaths within the remaining sample of 960 . Demographic and sleep characteristics, together with follow-up concordance levels, are shown across sleep duration categories in Table 1. The mean total sleep time was 7.1 hours (SD 1.5 hours), with most (52.9\%) participants reporting 6-8 hours sleep. At both the 4 and 8 years follow-ups, sleep duration categories among survivors showed a significant degree of concordance with those calculated at baseline (kappa $=0.16, p<0.001$; and kappa $=0.13, p<0.001$ respectively), with percentage agreement within categories ranging from $20-40 \%$ (Table 1). In analyses collapsing these categories into shorter ( $\leq 5$ hours), intermediate (6-8 hours) and longer (> 8 hours) sleep durations, percentage agreement at the 4-year (kappa $=0.29, p<0.001)$ and 8year $(\mathrm{kappa}=0.16, \mathrm{p}<0.001)$ follow-ups ranged from $40-60 \%$. Associations between sleep duration and measures of sleep quality (Table 1) were predominantly linear, with longer sleep times significantly associated with longer periods spent in bed $(F=326.1$; $d f=1,958$; $p<0.001)$, higher sleep efficiency $(F=934.1 ; d f=1,958 ; p<0.001)$, and a lower likelihood of reporting insomnia symptoms (chi-square $=58.21, \mathrm{df}=5, \mathrm{p}<0.001)$. Relationships between sleep duration and health and physical activity outcomes, on the other hand, were predominantly quadratic, with most approximating a U-shaped function (Table 2). Participants sleeping either more or less than 6-8 hours showed the lowest levels of overall physical activity (chi square $=42.4, \mathrm{df}=20, \mathrm{p}<0.01$ ), and the lowest levels of handgrip strength and general health (all $p<0.01$; Table 2). Depression showed a less consistent relationship with sleep duration, though high levels of clinical symptoms were associated with the shortest ( $\leq 4$ hours) sleep, while those with the longest sleep durations ( $>9$ hours) reported the lowest levels of depression (Table 2). A significant negative correlation was found between TIB as a continuous variable and CPA1 scores ( $r$ o $=-0.18 ; n=960$; $p<0.001$ ), though the correlation between TST and CPA1 scores and was not significant (rho $=0.03 ; n=960 ; p=0.33$. 
Hazard ratios $(\mathrm{HR})$ and confidence intervals $(\mathrm{Cl})$ for all-cause mortality from the Cox regression analyses are shown in Table 3. Adjusted only for age and gender, Model 1 shows that those who slept for $\geq 9$ hours had a significantly elevated risk of mortality over the 27-year follow-up period (HR: 1.4; 95\% Cl 1.08-1.83). This level of hazard associated with long sleep duration was only marginally attenuated when mental and physical health confounders were added in Model $2(\mathrm{HR}=1.37 ; 95 \% \mathrm{Cl}: 1.05-1.78)$. Within this model hypnotic usage was also associated with significant risk (HR: $1.24 ; 95 \% \mathrm{Cl}: 1.01-1.51$; Table 3). Following the addition of TIB category, physical activity level, handgrip strength and walking speed in Model 3, the risk associated with long ( $\geq 9$ hours) sleep duration was no longer significant (HR: 1.18; $95 \% \mathrm{Cl}: 0.85-1.63)$, while the lowest quintile for physical activity, and the slowest walking speed were both associated with significantly elevated mortality risk

(HR: 1.79; 95\% Cl: 1.40-2.30; HR: 1.41; 95\% Cl: 1.15-1.73 respectively; Table 3). Excluding deaths in the first 3 years after baseline, the association with mortality hazard remained significant for long sleep duration across models 1 and 2, and for the lowest physical activity quintile (HR: 046; 95\% Cl: 1.07-1.99) and very slow walking speed (HR: 1.33; 95\% Cl: 1.051.68) in Model 3 (see Table 4). Continuous hypnotic usage did not emerge as a significant mortality risk in any of the analyses omitting mortality in the first 3 years. Insomnia was not significantly associated with mortality in models using either the full follow-up data, or omitting mortality in the first 3 years.

\section{DISCUSSION}

The present findings from a nationally representative sample are strengthened by interviewer-assessed profiles of sleep quantity and quality, validated survey measures of mental and physical health, physical capacities, and physical activity levels, follow-up sleep assessments demonstrating significant stability in the reported sleep duration categories, and a large number of accurately recorded deaths. The proportions represented in the sleep duration categories (Table 1) are also comparable with those found in similarly aged samples (Castro-Costa et al, 2011; Chen et al, 2013), while the average sleep time of 7.1 
hours calculated for the whole sample falls between that reported for older people in Brazil (Castro-Costa et al, 2011) and Taiwan (Chen et al, 2013), 7.2 and 6.3 hours respectively. These results, therefore, offer a detailed and authoritative profile of sleep, activity and health at baseline, and a robust test of the influence of functional status on sleep duration-mortality relationships.

In general, increases in sleep duration were associated with superior sleep quality, with those in the longer $(7,8$ and $\geq 9$ hours) sleep duration categories reporting the shortest sleep latencies, the highest sleep efficiency scores, and the lowest levels of insomnia symptoms (Table 1). Indices of health and functional ability, on the other hand, showed a more u-shaped relationship with TST, with those in the $\leq 4$ hours category showing the highest levels of depression and physical health problems, and those in the $\geq 9$ hours category showing the lowest levels of activity. These findings accord with the results of Castro-Costa et al (2011) for physical and mental health, and Stranges et al (2008) and Bellavia et al (2014) for daytime physical activity. In addition, and over one of the longest follow-up periods yet reported for a random sample of older people, the present results align with earlier findings (e.g. Castro-Costa et al, 2011; Chen et al, 2013; Kurina et al, 2013; Bellavia, 2014) showing that longer (rather than shorter) sleep durations are more strongly associated with elevated all-cause mortality risk in models adjusted for mental and physical health status.

The unique contributions of the present study, however, are to show: 1) that long ( $\geq 9$ hours) sleepers are characterized by low levels of daily exercise, low muscle strength, and very slow walking speeds (all indicative of prefrailty/frailty); and 2) that two of these attributes (low levels of physical activity and very slow walking speed) are associated with elevated mortality risk even after controlling for long sleep duration, mental and physical health status, insomnia symptoms and hypnotic drug use. A reasonable inference to draw from these results is that indices of functional ability indicative of prefrail and frail states contribute to the phenomenon of long sleep duration-mortality relationships. Our analyses also confirm the supposition that these indices of functional status are inadequately captured (and controlled 
for) by conventional survey measures of health status. Nevertheless, the present results do appear to be at variance with Lee et al's (2017) recent finding that prefrailty and long sleep durations were significant but independent predictors of mortality. Several factors suggest these reported differences may principally reflect differing methodologies. Relative to the Lee et al (2017) study, our analyses: included an older population ( $\geq 53$ years $v \geq 65$ years); used a substantially longer follow-up period (4.7 years $v 27$ years); consequently captured more deaths (72 v 927); used more TST categories as covariates ( $3 v 6$ ); employed a different threshold for long sleep duration ( $\geq 8$ hours $v \geq 9$ hours); and controlled for TIB. That said, it should also be emphasized that the HRs for long sleep duration in the present Models 2 and 3 were quite similar, even though the latter failed to achieve significance. In conclusion, then, the present findings from the survival models (Table 3), together with health and activity profiles (Table 2) suggest a high degree of confounding among the TST and functional capacity covariates. While we would broadly agree with Kurina et al (2013) that ambiguities in the causal pathway from TST to mortality make it difficult to distinguish between confounders and mediators, the pattern of results shown here is consistent with the conclusion that very low levels of daytime activity, very slow walking speeds, and longer times spent in bed provide candidate factors plausibly contributing, directly or indirectly, to longer term mortality risk among those sleeping $\geq 9$ hours. Such a conclusion is also in agreement with Bellavia et al's (2014) suggestion that comorbidity with low physical activity might, at least in part, explain long sleep - mortality relationships. However, caution in interpreting the influence of the CPA1 in the survival models reported here is appropriate. In contrast to the findings of Bellavia et al (2014), which classified long sleep as $\geq 8$ hours, the present analyses found no significant interaction between physical activity and TST in predicting mortality.

Evidence of a reciprocal relationship between daily activity level and TIB was provided by the significant, though modest negative correlation between these variables (rho $=-0.18 ; n=$ 960; $p<0.001$ ) indicating diminishing daytime activity as TIB increased (though again, the TIB-CPA1 interaction term was not significant in predicting mortality). Evidence that TIB 
might independently compromise health is provided by the InChianti study (Stenholm et al, 2011) which found that both total sleep time and time in bed predicted accelerated decline in physical functioning among older people 3 and 6 years after baseline assessments. Taken together, therefore, the present results suggest that the activity and TIB profiles, and the mortality outcomes of those in the longest ( $\geq 9$ hours) sleep duration category, could be indicative of incipient frailty. The possibility also remains that the inactivity characteristic of frailty states may, in itself, attract the health risks associated with sedentary behavior.

Finally, several factors, arising both from the design of the present study, and the present outcomes, should be recognized as limitations. Designed at a time when clinical awareness of obstructive sleep apnea (OSA) was just developing in the UK (see, for example, Shapiro et al, 1981), the protocol did not include questions on snoring or daytime sleepiness which could have contributed to better characterizing the sleep symptoms reported here. While unequivocally a limitation, we concur with Cappuccio et al (2010, pp 590) who concluded that OSA-related mortality risk would most likely arise in obese shorter sleepers, and that “...adjustment for obesity or body mass index... would have corrected for this." While we are confident that the present findings extend earlier studies of subjectively reported nighttime sleep, the impact of total 24-hour sleep on mortality hazard, and the relationship of such risk to sleep obtained outwith the night-time sleep period remains to be investigated.

We also acknowledge the evident limitations of extrapolating from analyses performed on data collected from an earlier generation of older people. Reductions in physical activity levels, and increases in levels overweight/obesity seen in recent decades (Health and Social Care Information Centre, 2014) could have introduced generational or cohort effects which impacted the key variables reported here and, as a result, possibly limit the contemporary relevance of the findings. However, while these trends may have influenced the overall levels of, for example, the BMI and activity variables, it is less clear how secular change would impact relationships between these variables and health outcomes. That the 
relationships identified in this study accord with contemporary findings drawn from more recent cohorts supports the validity of the conclusions drawn. 


\section{ACKNOWLEDGEMENT}

We are pleased to acknowledge the important contributions of Daisy Johnson and lain Tomeny in managing and collating the NLSAA database.

\section{REFERENCES}

Afilalo, J., Alexander, K. P., Mack, M. J., Maurer, M. S., Green, P., Allen, L. A., et al. (2014).

Frailty Assessment in the Cardiovascular Care of Older Adults. Journal of the American College of Cardiology, 63(8), 747-762.

Armstrong, G. K., \& Morgan, K. Stability and change in levels of habitual physical activity in later life. Age and Ageing, 1998, 27, 17-23.

Aurora, R. N., Kim, J. S., Crainiceanu, C., O'hearn, D., \& Punjabi, N. M. (2016). Habitual sleep duration and all-cause mortality in a general community sample. Sleep, 39(11), 1903-1909.

Bassey, E. J. Longitudinal changes in selected physical capabilities: muscle strength, flexibility and body size. Age and Ageing, 1998, 27, 12-16.

Bath, P. A., \& Morgan, K. Customary physical activity and physical health outcomes in later life. Age and Ageing, 1998, 27, 29-34.

Bedford, A., Foulds, G. A., \& Sheffield, B. F. A new personal disturbance scale (DSSISAD). British Journal of Social and Clinical Psychology, 1976, 387-394.

Bellavia, A., Akerstedt, T., Bottai, M., Wolk, A., \& Orsini, N. Sleep Duration and Survival Percentiles Across Categories of Physical Activity. American Journal of Epidemiology, 2014, 179(4), 484-491. 
Cappuccio, F. P., D'Elia, L., Strazzullo, P., \& Miller, M. A. Sleep Duration and All-Cause Mortality: A Systematic Review and Meta-Analysis of Prospective Studies. Sleep, 2010, 33(5), 585-592.

Castro-Costa, E., Dewey, M. E., Ferri, C. P., Uchoa, E., Firmo, J. O. A., Rocha, F. L., et al. Association between sleep duration and all-cause mortality in old age: 9-year follow-up of the Bambui Cohort Study, Brazil. Journal of Sleep Research, 2011, 20(2), 303-310.

Chen, H.-C., Su, T.-P., \& Chou, P. (2013). A Nine-Year Follow-up Study of Sleep Patterns and Mortality in Community-Dwelling Older Adults in Taiwan. Sleep, 36(8), 1187-1198.

Clegg, A., Young, J., Iliffe, S., Rikkert, M. O., \& Rockwood, K. (2013). Frailty in elderly people. Lancet, 381(9868), 752-762.

Elias P., Halstead K., \& Prandy K. Computer assisted standard occupation coding. HMSO, London, 1993.

Ferrie, J. E., Shipley, M. J., Cappuccio, F. P., Brunner, E., Miller, M. A., Kumari, M., et al. (2007). A prospective study of change in sleep duration: Associations with mortality in the Whitehall II Cohort. Sleep, 30(12), 1659-1666.

Fried, L. P., Tangen, C. M., Walston, J., Newman, A. B., Hirsch, C., Gottdiener, J., et al. (2001). Frailty in older adults: Evidence for a phenotype. Journals of Gerontology Series a-Biological Sciences and Medical Sciences, 56(3), M146-M156.

Gabelle, A., Gutierrez, L. A., Jaussent, I., Navucet, S., Grasselli, C., Bennys, K., ... \& Vellas, B. (2017). Excessive sleepiness and longer nighttime in bed increase the risk of cognitive decline in frail elderly subjects: The MAPT-Sleep Study. Frontiers in aging neuroscience, 9,312 . 
Gallicchio, L., \& Kalesan, B. (2009). Sleep duration and mortality: a systematic review and meta-analysis. Journal of Sleep Research, 18(2), 148-158.

Grandner, M. A., \& Drummond, S. P. (2007). Who are the long sleepers? Towards an understanding of the mortality relationship. Sleep medicine reviews, 11(5), 341-360.

Hartescu, I., Morgan, K., \& Stevinson, C.D. (2015). Sleep Quality and Recommended Levels of Physical Activity in Older People. Journal of Aging and Physical Activity 24(2), 201-206.

Health and Social Care Information Centre (2014). Statistics on Obesity, Physical Activity and Diet: England 2014. Health and Social Care Information Centre, 2014. http://www.hscic.gov.uk/catalogue/PUB13648/Obes-phys-acti-diet-eng-2014-rep.pdf. Accessed May 2014.

Ikehara, S., Iso, H., Date, C., Kikuchi, S., Watanabe, Y., Wada, Y., et al. (2009). Association of sleep duration with mortality from cardiovascular disease and other causes for Japanese men and women: the JACC Study. Sleep, 32(3), 295-301.

Jike, M., Itani, O., Watanabe, N., Buysse, D. J., \& Kaneita, Y. (2017). Long sleep duration and health outcomes: A systematic review, meta-analysis and meta-regression. Sleep medicine reviews, 39, 25-36.

Kakizaki, M., Kuriyama, S., Nakaya, N., Sone, T., Nagai, M., Sugawara, Y., et al. (2013). Long sleep duration and cause-specific mortality according to physical function and selfrated health: the Ohsaki Cohort Study. Journal of Sleep Research, 22(2), 209-216.

Knutson, K. L., \& Turek, F. W. (2006). The U-shaped association between sleep and health: the 2 peaks do not mean the same thing. Sleep, 29(7), 878-879. 
Kripke, D. F., Garfinkel, L., Wingard, D. L., Klauber, M. R., \& Marler, M. R. (2002). Mortality associated with sleep duration and insomnia. Archives of General Psychiatry, 59(2).

Krueger, P. M., \& Friedman, E. M. (2009). Sleep Duration in the United States: A Crosssectional Population-based Study. American Journal of Epidemiology, 169(9), 10521063.

Kurina, L. M., McClintock, M. K., Chen, J. H., Waite, L. J., Thisted, R. A., \& Lauderdale, D. S. (2013). Sleep duration and all-cause mortality: a critical review of measurement and associations. Annals of Epidemiology, 23(6), 361-370.

Lee, J. S., Auyeung, T. W., Leung, J., Chan, D., Kwok, T., Woo, J., \& Wing, Y. K. (2014). Long sleep duration is associated with higher mortality in older people independent of frailty: a 5-year cohort study. Journal of the American Medical Directors Association, 15(9), 649-654.

Lee, W. J., Peng, L. N., Liang, C. K., Chiou, S. T., \& Chen, L. K. (2017). Long sleep duration, independent of frailty and chronic Inflammation, was associated with higher mortality: A national population-based study. Geriatrics \& gerontology international, 17(10), 1481-1487.

Lehmann, A. B., Bassey, E. J., Morgan, K., \& Dallosso, H. M. (1991). Normal values for weight, skeletal size and body-mass indexes in 890 men and women aged over 65 years. Clinical Nutrition, 10(1), 18-22.

Morgan, K. (1998). The Nottingham Longitudinal Study of Activity and Ageing: a methodological overview. Age and Ageing, 27, 5-11.

Morgan, K. (2003). Daytime activity and risk factors for late-life insomnia. Journal of Sleep Research, 12(3), 231-238. 
Patel, S. R., Ayas, N. T., Malhotra, M. R., White, D. P., Schernhammer, E. S., Speizer, F. E., et al. (2004). A prospective study of sleep duration and mortality risk in women. Sleep, 27(3), 440-444.

Patel, S. R., Malhotra, A., Gottlieb, D. J., White, D. P., \& Hu, F. B. (2006). Correlates of long sleep duration. Sleep, 29(7), 881-889.

Patel, S. R., Malhotra, A., Gao, X., Hu, F. B., Neuman, M. I., \& Fawzi, W. W. (2012). A prospective study of sleep duration and pneumonia risk in women. Sleep, 35(1), 97101.

Samitz, G., Egger, M., \& Zwahlen, M. (2011). Domains of physical activity and all-cause mortality: systematic review and dose-response meta-analysis of cohort studies. International Journal of Epidemiology, 40(5), 1382-1400.

Seixas, A., Henclewood, D. A., Williams, S. K., Jagannathan, R., Ramos, A., Zizi, F., \& Jean-Louis, G. (2018). Sleep duration and physical activity profiles associated with selfreported stroke in the United States: Application of Bayesian Belief Network Modeling techniques. Frontiers in neurology, 9, 534.

Shapiro, C. M., Catterall, J. R., Oswald, I., \& Flenley, D. C. (1981). Where are the British sleep apnea patients? Lancet, 2(8245), 523-523.

Stenholm, S., Kronholm, E., Bandinelli, S., Guralnik, J. M., \& Ferrucci, L. (2011). SelfReported Sleep Duration and Time in Bed as Predictors of Physical Function Decline: Results from the InCHIANTI Study. Sleep, 34(11), 1583-1593.

Stranges, S., Dorn, J. M., Shipley, M. J., Kandala, N. B., Trevisan, M., Miller, M. A., et al. (2008). Correlates of Short and Long Sleep Duration: A Cross-Cultural Comparison Between the United Kingdom and the United States The Whitehall II Study and the 
Western New York Health Study. American Journal of Epidemiology, 168(12), 13531364.

Thorp, A. A., Owen, N., Neuhaus, M., \& Dunstan, D. W. (2011). Sedentary Behaviors and Subsequent Health Outcomes in Adults A Systematic Review of Longitudinal Studies, 1996-2011. American Journal of Preventive Medicine, 41(2), 207-215.

World Health Organization 2010 Global Recommendations on Physical Activity for Health. Geneva, World Health Organization. ISBN 9789241599979. http://whqlibdoc.who.int/publications/2010/9789241599979 eng.pdf. Accessed September, 2013.

Youngstedt, S. D., \& Kripke, D. F. (2004). Long sleep and mortality: rationale for sleep restriction. Sleep Medicine Reviews, 8(3), 159-174. 
Table 1 Demographic and sleep characteristics at baseline by sleep duration

\begin{tabular}{|c|c|c|c|c|c|c|c|}
\hline & \multicolumn{7}{|c|}{ Sleep duration (hours) } \\
\hline & $\leq 4$ & 5 & 6 & 7 & 8 & $\geq 9$ & $P$ \\
\hline Total = 960: $n(\%)$ & $94(9.8)$ & $126(13.1)$ & $228(23.8)$ & $279(29.1)$ & $161(16.8)$ & $72(7.5)$ & \\
\hline \multicolumn{8}{|l|}{ Gender } \\
\hline Women: n (\%) & $66(11.3)$ & $87(14.9)$ & $133(22.7)$ & $154(26.3)$ & $100(17.1)$ & $45(7.7)$ & \\
\hline Men: $\mathrm{n}(\%)$ & $28(7.5)$ & $39(10.4)$ & $95(25.3)$ & $125(33.3)$ & $61(16.3)$ & $27(7.2)$ & $<0.05^{\dagger}$ \\
\hline Age: mean (median/range) & $76(75 / 29)$ & $75(74 / 29)$ & $74(74 / 28)$ & $75(75 / 30)$ & $76(75 / 26)$ & $77(77 / 33)$ & $0.03^{\ddagger}$ \\
\hline \multicolumn{8}{|l|}{ Social Class N (\%) } \\
\hline Professional/managerial & $12(8.1)$ & $15(10.1)$ & $42(28.4)$ & $46(31.1)$ & $24(16.2)$ & $9(6.1)$ & \\
\hline Skilled manual/non-manual & $57(10.0)$ & $87(15.2)$ & $125(21.9)$ & $157(27.5)$ & $100(17.5)$ & $45(7.9)$ & \\
\hline Semi-/unskilled manual & $25(10.4)$ & $24(10.0)$ & $61(25.3)$ & $76(31.5)$ & $37(15.4)$ & $18(7.5)$ & $0.45^{\dagger}$ \\
\hline Time in bed (TIB) hours: mean (SD) & $8.2(1.5)$ & $8.0(1.4)$ & $8.4(1.1)$ & $8.8(0.8)$ & $9.5(0.9)$ & 10. $8(1.1)$ & $<0.001^{*}$ \\
\hline Sleep Latency minutes: mean (SD) & $53(40.1)$ & $36.8(35.8)$ & $25.1(27.9)$ & $18.0(19.3)$ & $15.8(19.6)$ & $16.4(15.6)$ & $<0.001^{*}$ \\
\hline Sleep efficiency (SE): mean (SD) & $53.0(15.1)$ & $73.1(12.2)$ & $80.3(9.5)$ & $87.3(7.0)$ & $90.63(6.93)$ & $92.5(5.9)$ & $<0.001^{*}$ \\
\hline Prevalent insomnia symptoms: $\mathrm{n}$ & $45(22.8)$ & $28(14.2)$ & $48(24.4)$ & $42(21.3)$ & $23(11.7)$ & $11(5.6)$ & $<0.001^{\dagger}$ \\
\hline
\end{tabular}


Hypnotic use: $n(\%)$

Non-user

Occasional user

Continuous user

Survivors in same sleep duration

category at 4-year follow-up: $\mathrm{n}$

(column \%)

Survivors in same sleep duration

$10(22.7)$

$10(20.8)$

$33(32.7)$

$43(39.8)$

$16(25.4)$

$5(26.3)$

$<.001^{\S}$

(column \%)

Percentages are row values unless stated otherwise; SD, Standard Deviation; ${ }^{\dagger}$ significance of chi-square; ${ }^{\ddagger}$ Significance of Kruskal-Wallis 1-way ANOVA; §Significance of Cohen's kappa; *significance of regression F-value testing goodness of fit of linear model 
Table 2 Activity and health profiles at baseline by sleep duration

Sleep duration (hours)

\begin{tabular}{llllllll}
\hline$\leq 4$ & 5 & 6 & 7 & 8 & $\geq 9$ & $\mathrm{P}$
\end{tabular}

Customary Physical Activity Score

Quintiles: n (\%)

Low activity

$25(26.6) \quad 26(20.6) \quad 47(20.6) \quad 39(14.0) \quad 26(16.1) \quad 22(30.6)$

Medium/low activity

$23(24.5) \quad 21(16.7) \quad 41(18.0) \quad 46(16.5) \quad 40(24.8) \quad 25(34.7)$

Medium activity activity

$16(17.0) \quad 25(19.8) \quad 44(19.3) \quad 65(23.3) \quad 29(18.0) \quad 10(13.9)$

Medium/high activity

$15(16.0) \quad 27(21.4) \quad 43(18.9) \quad 68(24.4) \quad 35(21.7) \quad 8(11.1)$

High activity

Typical walking speed "very slow": n (\%)

$15(16.0) \quad 27(21.4) \quad 53(23.2) \quad 61(21.9) \quad 31(19.3) \quad 7(9.7) \quad 0.003^{\dagger}$

Maximum handgrip in newtons: mean (SD)

$19(20.4) \quad 26(20.6) \quad 44(19.5) \quad 44(15.8) \quad 37(23.0) \quad 30(42.3) \quad 0.001^{\dagger}$

$\begin{array}{lllllll}234.1 & 242.6 & 264.8 & 266.5 & 254.6 & 231.3 & <0.001 \ddagger\end{array}$

(101.8) (99.6) (109.3) (97.1) (101.5) (89.4)

Maximum handgrip in lowest quintile: $\mathrm{n}(\%)$

$27(28.7) \quad 23(19.0) \quad 38(17.4) \quad 44(16.1) \quad 35(23.3) \quad 18(27.3) \quad<0.001^{\ddagger}$

BMI: n (\%)

$$
\begin{aligned}
& <18.5 \text { - underweight } \\
& >18.5 \text { to }<25 \text { - normal }
\end{aligned}
$$$$
7(7.4) \quad 14(11.1) \quad 19(8.3) \quad 21(7.5) \quad 14(8.7) \quad 7(9.7)
$$$$
43(45.7) \quad 62(49.2) \quad 112(49.1) \quad 157(56.3) \quad 76(47.2) \quad 41(56.9)
$$ 
$\geq 25$ - overweight

Health Index: mean (SD)§

Survey classification of

depression: $\mathrm{n}$ (column \%)

$\begin{array}{lllllll}44(46.8) & 50(39.7) & 97(42.5) & 101(36.2) & 71(44.1) & 24(33.3) & 0.56^{\dagger} \\ 5.4(2.6) & 4.6(2.8) & 4.4(2.7) & 4.0(2.5) & 4.1(2.5) & 5.0(2.6) & <0.01^{\ddagger} \\ 18(19.1) & 10(7.9) & 21(9.2) & 13(4.7) & 18(11.2) & 9(12.5) & 0.001^{\dagger}\end{array}$

$44(46.8) \quad 50(39.7) \quad 97(42.5) \quad 101(36.2) \quad 71(44.1) \quad 24(33.3) \quad 0.56^{\dagger}$

Percentages are row values; SD, Standard Deviation; ${ }^{\dagger}$ Significance of chi-square; ${ }^{\ddagger}$ Significance of regression F-value testing goodness of fit of quadratic model; \$Higher scores indicate greater health burden 
Table 3 Hazard ratios (HR) from Cox regression models*: all-cause mortality at 27-year follow-up

\begin{tabular}{|c|c|c|c|c|c|c|c|}
\hline \multirow{2}{*}{$\begin{array}{l}\text { Covariate } \\
\text { Sleep duration }\end{array}$} & \multirow{2}{*}{$\begin{array}{l}\text { No. of } \\
\text { deaths }\end{array}$} & \multicolumn{2}{|l|}{ Model 1} & \multicolumn{2}{|l|}{ Model 2} & \multicolumn{2}{|l|}{ Model 3} \\
\hline & & $\mathrm{HR}(95 \% \mathrm{Cl})$ & $\mathrm{P}$ & $\mathrm{HR}(95 \% \mathrm{Cl})$ & $P$ & $\mathrm{HR}(95 \% \mathrm{Cl})$ & $P$ \\
\hline$\leq 4$ hours & 92 & $1.02(0.80-1.29)$ & 0.89 & $1.02(0.80-1.31)$ & 0.87 & $1.08(0.83-1.4)$ & 0.58 \\
\hline 5 hours & 123 & $1.04(0.84-1.29)$ & 0.72 & $1.02(0.82-1.27)$ & 0.85 & $1.01(0.79-1.29)$ & 0.91 \\
\hline 6 hours & 221 & $1.00(0.84-1.20)$ & 0.97 & $1.00(0.83-1.20)$ & 0.97 & $0.97(0.80-1.18)$ & 0.77 \\
\hline 7 hours & 270 & Reference & & Reference & & Reference & \\
\hline 8 hours & 150 & $0.89(0.73-1.09)$ & 0.26 & $0.90(0.74-1.11)$ & 0.32 & $0.88(0.71-1.10)$ & 0.27 \\
\hline$\geq 9$ hours & 71 & $1.40(1.08-1.83)$ & 0.01 & $1.37(1.05-1.78)$ & 0.02 & $1.18(0.85-1.63)$ & 0.32 \\
\hline \multicolumn{8}{|l|}{ Hypnotic use } \\
\hline Non-user & 720 & & & Reference & & Reference & \\
\hline Occasional user & 76 & & & $0.87(0.69-1.13)$ & 0.33 & $0.83(0.64-1.07)$ & 0.15 \\
\hline Continuous user & 131 & & & $1.24(1.01-1.51)$ & 0.04 & $1.19(0.96-1.47)$ & 0.11 \\
\hline \multicolumn{8}{|l|}{ Activity level } \\
\hline Low & 182 & & & & & $1.79(1.40-2.30)$ & $<0.01$ \\
\hline Medium low & 190 & & & & & $1.16(0.92-1.46)$ & 0.46 \\
\hline
\end{tabular}




\begin{tabular}{|c|c|c|c|}
\hline Medium & 182 & $1.14(0.91-1.41)$ & 0.68 \\
\hline Medium & 187 & $1.07(0.86-1.34)$ & 0.58 \\
\hline \multicolumn{4}{|l|}{ high } \\
\hline High & 186 & Reference & \\
\hline \multicolumn{4}{|l|}{ ime in bed } \\
\hline$\leq 6$ hours & 76 & $0.99(0.74-1.31)$ & 0.91 \\
\hline 7 hours & 189 & $0.98(0.81-1.19)$ & 0.84 \\
\hline 8 hours & 323 & Reference & \\
\hline 9 hours & 208 & $1.19(0.98-1.43)$ & 0.08 \\
\hline$\geq 10$ hours & 100 & $1.03(0.78-1.35)$ & 0.86 \\
\hline \multicolumn{4}{|l|}{$\begin{array}{l}\text { Handgrip } \\
\text { Strength }\end{array}$} \\
\hline $\begin{array}{l}\text { Lowest quintile } \\
\text { Nalking speed }\end{array}$ & 180 & $1.05(0.87-1.26)$ & 0.62 \\
\hline Very slow & 173 & $1.41(1.15-1.73)$ & 0.01 \\
\hline
\end{tabular}

${ }^{*}$ Covariates for Model 1: age and gender; Model 2: as Model 1 plus social class, hypnotic drug use, insomnia symptoms, physical health, symptoms of depression, BMI, and smoking status; Model 3: as Model 2 plus physical activity quintile; very slow walking speed; frail handgrip strength; and TIB category. 
Table 4 Hazard ratios (HR) from Cox regression models* excluding deaths in the first 3 years post baseline: all-cause mortality at 27-year follow-up

\begin{tabular}{|c|c|c|c|c|c|c|c|}
\hline Covariate & $\begin{array}{l}\text { No. of } \\
\text { deaths }\end{array}$ & Model 1 & & Model 2 & & Model 3 & \\
\hline \multicolumn{8}{|l|}{ Sleep duration } \\
\hline$\leq 4$ hours & 72 & $0.98(0.75-1.28)$ & 0.9 & $0.98(0.74-1.29)$ & 0.88 & $1.04(0.78-1.39)$ & 0.79 \\
\hline 5 hours & 106 & $1.12(0.89-1.42)$ & 0.35 & $1.11(0.88-1.41)$ & 0.39 & $1.11(0.85-1.45)$ & 0.43 \\
\hline 6 hours & 182 & $1.01(0.83-1.23)$ & 0.67 & $1.02(0.83-1.25)$ & 0.86 & $0.99(0.80-1.23)$ & 0.93 \\
\hline 7 hours & 218 & Reference & & Reference & & Reference & \\
\hline 8 hours & 118 & $0.85(0.68-1.07)$ & 0.16 & $0.87(0.67-1.10)$ & 0.23 & $0.87(0.67-1.11)$ & 0.26 \\
\hline$\geq 9$ hours & 51 & $1.37(1.01-1.87)$ & 0.04 & $1.40(1.00-1.86)$ & 0.05 & $1.29(0.89-1.86)$ & 0.18 \\
\hline \multicolumn{8}{|l|}{ Activity level } \\
\hline Low & 122 & & & & & $1.46(1.07-1.99)$ & 0.02 \\
\hline Medium low & 160 & & & & & $1.09(0.83-1.41)$ & 0.54 \\
\hline Medium & 161 & & & & & $1.08(0.84-1.37)$ & 0.57 \\
\hline Medium high & 169 & & & & & $1.08(0.85-1.38)$ & 0.51 \\
\hline High & 168 & & & & & Reference & \\
\hline \multicolumn{8}{|l|}{ Walking speed } \\
\hline Very low & 116 & & & & & $1.33(1.05-1.68)$ & 0.02 \\
\hline
\end{tabular}


${ }^{*}$ Covariates for Model 1: age and gender; Model 2: as Model 1 plus social class, hypnotic drug use, insomnia symptoms, physical health, symptoms depression, BMI, and smoking status; Model 3: as Model 2 plus physical activity quintile; very slow walking speed; frail handgrip strength; and TIB category. 